\title{
POWER MAPS AND PRINCIPAL BUNDLES
}

\section{J. L. NOAKES}

ABSTRACT. Let $G$ be a path connected topological group. We investigate the integers $m$ for which the $m$ th power map on $G$ extends to an overmap of principal $G$-bundles.

This, our third note on the subject of fibre-preserving maps (called overmaps) succeeds [5], [6]. I wish to thank Professor I. M. James who supervised [4], for encouragement, and for considerable help with the exposition.

1. Statement of results. Let $G$ be a path connected topological group, and let $P, P^{\prime}$ be principal $G$-bundles over a path connected pointed base $(B, b)$. An overmap $P \rightarrow P^{\prime}$ is of power $m$ when its restriction to fibres over $b$ is the $m$ th power map. By subtracting overmaps we see that there is an integer $\alpha(P) \geqslant 0$ such that there is an overmap $P \rightarrow B \times G$ of power $m$ if and only if $m$ is divisible by $\alpha(P)$. Evidently $P$ is trivial if and only if $\alpha(P)=1$.

THEOREM 1. Suppose that there is an overmap $P \rightarrow P^{\prime}$ of power $n$. Then there is an overmap $P \rightarrow P^{\prime}$ of power $m$ if and only if $m \equiv n \bmod \alpha(P)$.

By means of the $G$-map $k_{m}^{\prime}: G \times G \rightarrow G$ given by $k_{m}^{\prime}(g, h)=h\left(g^{-1} h\right)^{m-1}$, the arguments of [5] prove a version of Theorem 1. However, it is better to prove Theorem 1 as follows.

Denote the reverse $G$-action $[8,8.11]$ on $P^{\prime}$ by

$$
j: P^{\prime} \times{ }_{B}(B \times G)=P^{\prime} \times G \rightarrow P^{\prime},
$$

and let $f: P \rightarrow P^{\prime}$ be an overmap of power $n$. Given an overmap $e: P \rightarrow B \times$ $G$, let $e^{+}: P \rightarrow P^{\prime}$ be $f \times{ }_{B} e: P \rightarrow P^{\prime} \times_{B}(B \times G)$ followed by $j$.

Let $k$ be inverse to the bundle equivalence

$$
p_{1} \times_{B} j: P^{\prime} \times{ }_{B}(B \times G) \rightarrow P^{\prime} \times{ }_{B} P^{\prime}
$$

where $p_{i}$ means projection to the $i$ th factor. Given an overmap $d: P \rightarrow P^{\prime}$, let $d^{-}: P \rightarrow B \times G$ be $f \times{ }_{B} d: P \rightarrow P^{\prime} \times{ }_{B} P^{\prime}$ followed by $p_{2} k$. Since $e^{+-}=e$, $d^{-+}=d$, we have a bijection between overmaps $P \rightarrow B \times G$ of power $m$ and overmaps $P \rightarrow P^{\prime}$ of power $m+n$. This proves Theorem 1 . From now on we suppose that $B, G$ are finite $C W$-complexes.

Received by the editors August 13, 1975 and, in revised form, July 12, 1976.

AMS (MOS) subject classifications (1970). Primary 55F 10. 
THEOREM 2. Suppose that there is an overmap $P^{\prime} \rightarrow P$ of power $n$. Then there is an overmap $P \rightarrow P^{\prime}$ of power $m$ if and only if

$$
m n \equiv 1 \bmod \text { l.c.m. }\left(\alpha(P), \alpha\left(P^{\prime}\right)\right) \text {. }
$$

For example let $P$ be induced from the Hopf bundle $S^{7} \rightarrow S^{4}$ (which we regard as a principal $S^{3}$-bundle) by the map $S^{3} \cup_{r} e^{4} \rightarrow S^{4}$ collapsing $S^{3}$. By [4, II.4.2], $\alpha(P)$ is $|r|$ or $2|r|$, and if $r$ is odd or if $r$ is divisible by 8 then $\alpha(P)=|r|$.

Let $H$ be another connected topological group that is a finite $\mathrm{CW}$-complex, and let $Q$ be a principal $H$-bundle over $B$.

THEOREM 3. The primes that divide $\alpha\left(P \times_{B} Q\right)$ are precisely those that divide $\alpha(P)$ or $\alpha(Q)$.

THEOREM 4. We have $\alpha(P)$ positive if and only if the rational cohomology characteristic classes of $P$ are zero.

For example if $B$ is a sphere of odd dimension, or a real projective space, or a generalised lens space, then $\alpha(P)$ is positive. Hence in these cases there are overmaps $P \rightarrow P$ of powers greater than 1 . However if $P$ is associated with the tangent bundle to $S^{2 q}(q>0)$ then $\alpha(P)=0$. In this case all overmaps $P \rightarrow P$ that restrict to power maps are of power 1 .

2. Function space bundles. We work in the category of compactly generated spaces [9]. Given $f: F \rightarrow F^{\prime}$, let $\mathcal{S}_{f}$ be the space of maps homotopic to $f$. Let $G^{\prime}$ be a path connected topological group and let $F, F^{\prime}$ be $G^{\prime}$-spaces. Then a $G^{\prime}$-action '*' on $\mathcal{G}_{f}$ is given by $(g * e)(x)=g \cdot\left(e\left(g^{-1} \cdot x\right)\right)$. Here $g \in G^{\prime}$, $e \in \mathcal{G}_{f}, x \in F$.

Let $R$ be a principal $G^{\prime}$-bundle over a path connected pointed base $(B, b)$. Formation of associated bundles defines a functor (which we also denote by $R$ ) from the category of $G^{\prime}$-spaces and $G^{\prime}$-maps to the category of compactly generated overspaces of $B$ and overmaps.

Given $q: Y \rightarrow B$ with nonempty fibre $Z$, let $\Re Y$ be the space maps $F \rightarrow Y$ whose composites with $q$ are constant. Given $f: F \rightarrow Z$, let $\mathfrak{\pi}_{f} Y$ be the path component in $\mathfrak{N} Y$ of $f$ followed by the inclusion of $Z$ in $Y$. Then $\left(\mathfrak{R} Y, \mathfrak{R}_{f} Y\right)$ is a pair of overspaces where $\Re q$ : $\Re Y \rightarrow B$ is given by $(\Re q)(h)=h(x)$. Here $h \in \Re Y, x \in F$. Composition with an overmap $k: Y \rightarrow Y^{\prime}$ defines an overmap $\Re k: \mathfrak{N} Y \rightarrow \mathfrak{N} Y^{\prime}$. In this way $\Re$ is a functor.

Choose for $R$ a coordinate cover $\left\{U_{i}\right\}_{i \in J}$ of $B$ and coordinate transformations $g_{i j}: U_{i} \cap U_{j} \rightarrow G^{\prime}$. Take the discrete topology on $J$, and let $T$ be the subspace of $\Re_{f} Y \times J$ consisting of pairs $(h, i)$ for which $\left(\Re_{q} q\right)(h) \in U_{i}$. Let $\Re_{f}^{R} Y$ be the space obtained from $T$ by identifying $(h, i),\left(h^{\prime}, j\right)$ when both the following conditions are met:

(1) $(\Re q)(h)=(\Re q)\left(h^{\prime}\right)=b^{\prime}$ say.

(2) $h(x)=h^{\prime}\left(g_{j i}\left(b^{\prime}\right) \cdot x\right)$ for all $x \in F$.

Then $\mathfrak{T}_{f}^{R} Y$ is an overspace of $B$, where $\mathfrak{\pi}^{R} q$ : $\mathfrak{\pi}_{f}^{R} Y \rightarrow B$ is given by 
$\left(\Re^{R} q\right)[h, i]=(\Re q)(h)$. Here $(h, i) \in T$. Composition with an overmap $k: Y \rightarrow Y^{\prime}$ defines an overmap $\pi^{R} k: \pi_{f}^{R} Y \rightarrow \pi_{g f}^{R} Y^{\prime}$ where $g$ is the restriction of $k$ to fibres. If $Y$ is a Serre fibre space and if $F$ is a CW-complex then, as in [4, I, §2], $\Re_{f}^{R} Y$ is a Serre fibre space. The following assertions are easy to verify.

(3) There is a natural homeomorphism from the space of extensions of $f$ from fibres to overmaps $R F \rightarrow Y$ to the space of cross-sections $s$ of $\pi_{f}^{R} Y$ for which $s(b)=f$.

(4) If $Y=R F^{\prime}$ then $\Re_{f}^{R} Y$ is homeomorphic by an overmap with $R \mathcal{S}_{f}$.

3. Localization. We work in the category of pointed compactly generated spaces, and in the category of pointed overspaces of $B$. Let $E, E^{\prime}$ be Serre fibre spaces over $B$ with nilpotent fibres [1, II.4.3] $F, F^{\prime}$. Let $\{M, N\}$ be a partition of the primes.

By the fibrewise localization $\dot{E}_{M}$ at $M$ of $E$ we mean the same as in [1, I $\S 8$, $\mathrm{V} \S 4]$, except that we work with topological spaces in place of simplicial sets. We denote the localizing overmap $E \rightarrow \dot{E}_{M}$ by $\dot{\varepsilon}_{M}$, and its restriction $F \rightarrow F_{M}$ to fibres by $\varepsilon_{M}$. If an overmap $f: E \rightarrow E^{\prime}$ restricts to $h$ on fibres, we denote its fibrewise localization by $\dot{f}_{M}$, and we denote the localization of $h$ by $h_{M}$. In this context it is customary to refer to 0 as a prime, and to talk of the fibrewise localization $\dot{E}_{0}$ of $E$ at 0 . I say that 0 is not a prime, and I talk instead of the fibrewise localization $\dot{E}_{\varnothing}$ of $E$ at the empty set.

In the situation of $\$ 1$ we prove the following result.

LEMMA 1. If there is an overmap $f: P \rightarrow P$ of power $n \neq 1$, and if $N$ contains only primes that divide $n$, then $\dot{P}_{N}$ has a cross-section.

For this it suffices to prove that $\dot{P}_{N} \mid B^{r}$ has a cross-section for all $r \geqslant 1$. Here $B^{r}$ is the $r$-skeleton of the CW-complex $B$. But $G_{N}$ is path connected and so $\dot{P}_{N} \mid B^{1}$ has a cross-section. Suppose inductively that, for some $q \geqslant 1, \dot{P}_{N} \mid B^{q}$ has a cross-section $s$, and let $c(s) \in H^{q+1}\left(B ; \pi_{q} \dot{G}_{N}\right)$ be the obstruction to extending $s \mid B^{q-1}$ to a cross-section of $\dot{P}_{N} \mid B^{q+1}$.

Consider the fibrewise localization $\dot{j}_{N}: \dot{P}_{N} \times_{B}(B \dot{\times} G)_{N} \rightarrow \dot{P}_{N}$ of the reverse $G$-action on $P$. Since

$$
\dot{j}_{N} \times_{B} p_{1}: \dot{P}_{N} \times_{B}(B \dot{\times} G)_{N} \rightarrow \dot{P}_{N} \times_{B} \dot{P}_{N}
$$

is a weak equivalence over $B$, there is a cross-section $s^{\prime}$ of $(B \dot{\times} G)_{N} \mid B^{q}$ such that $\left(j_{N} \times_{B} p_{1}\right)\left(s \times_{B} s^{\prime}\right)$ is homotopic through cross-sections to $s \times{ }_{B} \dot{f}_{N} s$, by $[3,3.2]$. Therefore $c\left(\dot{f}_{N} s\right)=c(s)+c\left(s^{\prime}\right)$.

By $[8,5.8 .13]$ there is a weak equivalence $G_{\varnothing} \rightarrow K$ where $K$ is a finite product of Eilenberg-Mac Lane groups $K(\mathbf{Q}, 2 t+1)$, and so $s^{\prime} \mid B^{q-1}$ followed by $\dot{\varepsilon}_{\varnothing}:(B \dot{\times} G)_{N} \rightarrow(B \dot{\times} G)_{\varnothing}$ extends to a cross-section of $(B \dot{\times} G)_{\varnothing} \mid B^{q+1}$. Therefore $\varepsilon_{\varnothing * *} c\left(s^{\prime}\right)=0$ where $\varepsilon_{\varnothing * *}: H^{q+1}\left(B ; \pi_{q} G_{N}\right) \rightarrow H^{q+1}\left(B ; \pi_{q} G_{\varnothing}\right)$ is induced by $\varepsilon_{\varnothing *}: \pi_{q} G_{N} \rightarrow \pi_{q} G_{\varnothing}$ on coefficients.

But $H^{q+1}\left(B ; \pi_{q} G_{N}\right)$ is a finitely generated $\mathbf{Z}_{N}$-module and so $n^{l} c\left(s^{\prime}\right)=0$ for some $l$. We have the following identities. 


$$
\begin{aligned}
& n c\left(\dot{f}_{N}^{l} s\right)=n c\left(\dot{f}_{N}^{l} s\right)=n^{l}\left(c(s)+c\left(s^{\prime}\right)\right), \\
& n^{l}\left(c(s)+c\left(s^{\prime}\right)\right)=n^{l} c(s)=c\left(\dot{f}_{N}^{l} s\right)
\end{aligned}
$$

These imply that $(n-1) c\left(\dot{f}_{N}^{l} s\right)=0$ and, since multiplication by $n-1$ is an automorphism of $\pi_{q} G_{N}, c\left(\dot{f}_{N}^{l} s\right)=0$. Hence $\dot{f}_{N}^{l} s \mid B^{q-1}$ extends to a crosssection of $\dot{P}_{N} \mid B^{q+1}$. This completes the induction, and the proof of Lemma 1.

4. Proof of Theorem 2. We regard $R=P \times_{B} P^{\prime}$ as a principal $G \times G$ bundle, and we denote $G$ by $F, F^{\prime}$ according to $G \times G$ acts by means of projection to the first, second factor. Let $s_{m}: G \rightarrow G$ be the $m$ th power map, and denote $\mathcal{G}_{s_{m}}\left(F, F^{\prime}\right)$ by $\mathcal{G}_{m}$.

LEMma 1. If $\dot{P}_{N}, \dot{P}_{N^{\prime}}$ have cross-sections then $\left(R \dot{\mathcal{G}}_{m}\right)_{N}$ has a cross-section for all $m$.

To prove this note first that by means of the reverse $G$-actions on $P, P^{\prime}$ the given cross-sections produce weak equivalences

$$
w:(B \dot{\times} G)_{N} \rightarrow \dot{P}_{N}, \quad w^{\prime}:(B \dot{\times} G)_{N} \rightarrow \dot{P}_{N}^{\prime},
$$

over $B$. By $[3,3.2]$ applied to the weak equivalence

$$
\mathfrak{T}^{R} w: \mathfrak{M}_{e_{N}}^{R}(B \dot{\times} G)_{N} \rightarrow \Re_{e_{N}}^{R} \dot{P}_{N},
$$

and by (2.3), there is an overmap $f: P \rightarrow(B \dot{\times} G)_{N}$ such that wf is homotopic through overmaps to $\dot{\varepsilon}_{N}$. Corresponding under (2.3) to

$$
P \stackrel{f}{\longrightarrow}(B \dot{\times} G)_{N} \stackrel{\left(1 \dot{\times} s_{m}\right)_{N}}{\longrightarrow}(B \dot{\times} G)_{N}
$$

there is a cross-section of $\mathfrak{N}_{\left(s_{m}\right)_{N} \varepsilon_{N}}^{R}(B \dot{\times} G)_{N}$. Composing this cross-section with $\mathfrak{R}^{R} w^{\prime}$, we get a cross-section $s$ of $\mathfrak{T}_{\left(s_{m}\right)_{N} e_{N}}^{R} \dot{P}_{N}^{\prime}$.

The fiberwise localization at $N$ of $\mathfrak{T}_{\varepsilon_{N}}^{R}: \mathfrak{M}_{s_{m}}^{R} P^{\prime} \rightarrow \mathfrak{R}_{\varepsilon_{N} s_{m}}^{R} \dot{P}_{N}^{\prime}$ is a weak equivalence. Therefore, by $[3,3.2]$ and since $\varepsilon_{N} s_{m}=\left(s_{m}\right)_{N} \varepsilon_{N},\left(\Re_{s_{m}}^{R^{\prime}} P^{\prime}\right)_{N}$ has a cross-section. But by (2.4) $\mathfrak{T}_{s_{m}}^{R} P^{\prime}=R \mathcal{G}_{m}$, and this completes the proof of Lemma 1.

LEMMA 2. If there are overmaps $P \rightarrow P, P^{\prime} \rightarrow P^{\prime}, P^{\prime} \rightarrow P$ of powers $m n, m n$, $n$, then there is an overmap $P \rightarrow P^{\prime}$ of power $m$.

To prove this note first that if $m n=0$ then $P, P^{\prime}$ are trivial by (1.1). If $m=n=1$ then Lemma 2 holds trivially, and if $m=n=-1$ then Lemma 2 is a consequence of $[2,6.3]$. Suppose therefore that $m n \neq 0,1$ and let $N$ be the set of primes that divide $n$.

By (3.1) $\dot{P}_{N}, \dot{P}_{N}^{\prime}$ have cross-sections, and so $\left(R \dot{\mathcal{S}}_{m}\right)_{N}$ has a cross-section by Lemma 1. By (2.3), (2.4), [6, 4.1], $\left(R \dot{\mathcal{G}}_{m}\right)_{M}$ has a cross-section. Hence, and by $[6,3.3], R \mathcal{S}_{m}$ has a cross-section. Therefore, by (2.3), (2.4) again, there is an overmap $P \rightarrow P^{\prime}$ of power $m$. This proves Lemma 2 .

5. Primes dividing $\alpha(P)$. In the situation of $\S 1$ we prove the following result.

Proposition 1. The primes $p$ that divide $\alpha(P)$ are precisely those for which the 
localization $\dot{P}_{p}$ does not have a cross-section.

In order to prove Proposition 1 we require a lemma. For each positive integer $i$ let $N(i)$ be a set of primes. Let $N=\cup_{i} N(i)$.

LEMMA 2. If, for each $i, \dot{P}_{N(i)}$ has a cross-section then $\dot{P}_{N}$ has a cross-section.

To prove Lemma 2 consider the commuting diagram

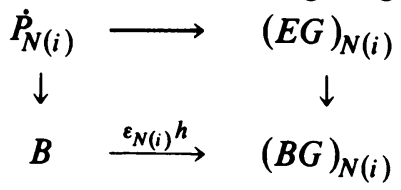

where $E G \rightarrow B G$ is a universal principal $G$-bundle and $h: B \rightarrow B G$ classifies $P$. Since $\dot{P}_{N(i)}$ has a cross-section and $E G$ is contractible we have $\varepsilon_{N(i)} h \simeq$. Therefore by [1, V. 6.2] $\varepsilon_{N} h \simeq$, and so $\dot{P}_{N}$ has a cross-section. This proves Lemma 2.

Let $N$ be the set of primes $p$ such that $\dot{P}_{p}$ has a cross-section. Then $\dot{P}_{N}$ has a cross-section by Lemma 2 . In the situation of $\S 4$ let $P^{\prime}$ be trivial. Then by (4.1) $\left(R \dot{\mathcal{G}}_{m}\right)_{N}$ has a cross-section for all $m$.

By obstruction theory either $M$ is empty or there is a product $m$ of primes in $M$ such that $\left(P \dot{\mathcal{G}}_{m}\right)_{M}$ has a cross-section. If $M$ is empty then $P$ has a crosssection and $\alpha(P)=1$. In the second case $R \mathcal{G}_{m}$ has a cross-section by $[6,3.3]$, and there is an overmap $P \rightarrow B \times G$ of power $m$ by (2.3), (2.4). Let $M^{\prime}, N^{\prime}$ be the sets of primes that divide, do not divide $\alpha(P)$. Then we have shown that $M^{\prime} \subseteq M$.

Now let $f: P \rightarrow B \times G$ be an overmap of power $\alpha(P)$. Since $\dot{f}_{N^{\prime}}$ is a weak equivalence. $\dot{P}_{N^{\prime}}$ has a cross-section. Hence $N^{\prime} \subseteq N$. This completes the proof of Proposition 1.

As a consequence of Proposition 1 we have (1.3). Also $\dot{P}_{\varnothing}$ has a crosssection if and only if the rational cohomology characteristic classes of $P$ are zero. Therefore Proposition 1 also implies (1.4).

\section{REFERENCES}

1. A. K. Bousfield and D. M. Kan, Homotopy limits, completions and localizations, Lecture Notes in Math., vol. 304, Springer-Verlag, Berlin and New York, 1972. MR 51 \# 1825.

2. A. Dold, Partitions of unity in the theory of fibrations, Ann. of Math. (2) 78 (1963), 223-255. MR 27 \# 5264.

3. I. M. James, Overhomotopy theory, Symposia Mathematica, Vol. IV (INDAM, Rome, 1968/69), Academic Press, London, 1970, pp. 219-229. MR 43 \#1183.

4. J. L. Noakes, Some topics in homotopy theory, Ph.D. thesis, Univ. of Oxford, 1974, pp. 1-63.

5. - Symmetric overmaps, Proc. Amer. Math. Soc. (to appear).

6. - Unstable J-invariants, Quart. J. Math. Oxford Ser. (2) 27 (1976), 51-57.

7. E. H. Spanier, Algebraic topology, McGraw-Hill, New York, 1966. MR 35 \# 1007.

8. N. E. Steenrod, The topology of fibre bundles, Princeton Univ. Press, Princeton, N.J., 1951. MR 12, 522.

9. - A convenient category of topological spaces, Michigan J. Math. 14 (1967), 133-152. MR 35 \# 970.

Department of Mathematics, University of Western Australia, Nedlands, W.A. 6009, Australia 